\title{
60-MHz wavelength-encoded tomography (WET)
}

\author{
Bowen Li, ${ }^{1}$ Chi Zhang, ${ }^{2}$ Sisi Tan, ${ }^{1}$ Xiaoming Wei, ${ }^{1}$ Yiqing Xu ${ }^{1}$, Kevin K. Tsia ${ }^{1}$ and Kenneth K. Y. Wong ${ }^{1, *}$ \\ ${ }^{1}$ Department of Electrical and Electronic Engineering, The University of Hong Kong, Pokfulam Road, Hong Kong. \\ 2 Wuhan National Laboratory for Optoelectronics \& School of Optical and Electronic Information, \\ Huazhong University of Science and Technology, Wuhan 430074, China \\ *kywong@eee.hku.hk
}

\begin{abstract}
Wavelength-encoded tomography (WET) is upgraded to a triple-time-lens system to perform ultrafast cross-sectional imaging through $68.4 \mathrm{x}$-temporal magnification. $60-\mathrm{MHz} \mathrm{A}$-scan rate is demonstrated by imaging a glass sample with $180-\mu \mathrm{m}$ axial resolution.

OCIS codes: (110.6955) Tomographic imaging; (300.6300) Spectroscopy, Fourier transforms; (110.6915) Time imaging
\end{abstract}

\section{Introduction}

Tomographic imaging has found its wide application in biological science owing to its non-invasive cross-sectional detection capability. So far, various tomography modalities have been developed with diverse specialties. Optical coherence tomography (OCT) [1], for example, is popular for its fine resolution and moderate imaging depth. However, its interferometry-based detection method as well as the requirement for data post-processing set the inherent limits on the operation speed. On the other hand, a novel tomographic technique termed as wavelengthencoded tomography (WET) [2] was introduced recently, which is made of two time-lenses with opposite focal group delay dispersion (GDD). Depth information (time-delay) is directly converted to wavelength difference through optical Fourier transform. As the requirement for data processing is greatly relaxed, the WET system has the potential to achieve much faster speed than other tomographic techniques. However, as a proof-of-concept demonstration, only an optical spectrum analyzer (OSA) was utilized to detect the WET signal and the A-scan rate was limited to $5 \mathrm{~Hz}$, which rendered the system unpractical for real tomography.

Meanwhile, we have also proposed an ultrafast spectrum resolving modality, termed parametric spectro-temporal analyzer (PASTA) [3]. The system consists of a time-lens with following output dispersion and performs wavelength-to-time mapping through temporal focusing. In this paper, we integrate the PASTA into the WET to build a triple-time-lens tomography system. The PASTA-based WET performs depth (time-delay)-wavelength-time mapping and is essentially a temporal-magnification system with a magnification factor of $68.4 \mathrm{x}$. Even though temporal magnification has been well explored for ultrafast waveform characterization [4,5], this is the first time this technique is applied to tomography. As the depth information can be directly measured in the time domain, $60 \mathrm{MHz}$ A-scan rate is demonstrated, which is 12-million times faster than the first version of WET.

\section{Experiment and results}

The schematic ray diagram as well as the simplified setup of the PASTA-based WET system is shown in Fig. 1. The WET system consists of two four-wave mixing (FWM) based time-lenses with opposite focal GDDs $\left(\Phi_{I}\right.$ and $\left.-\Phi_{l}\right)$. With a continuous wave $(\mathrm{CW})$ input, a chirped pulse is generated by the first time-lens, which is illuminated onto the sample and reflected back with different delays. The depth information is encoded onto the timing of the chirped pulse, and will be mapped to the wavelength by the second time-lens through optical Fourier transform. The PASTA system, which is essentially a FWM time-lens with corresponding dispersion, is integrated after the WET system. It performs another optical Fourier transform to map the wavelength-encoded depth information to the time domain so that we can directly acquire the cross-sectional image using real-time oscilloscope. As a whole, the system magnifies the small time delays generated by the sample reflection to a much larger time separation so that the oscilloscope can resolve.

(a)

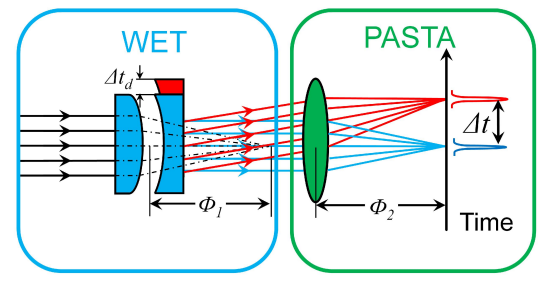

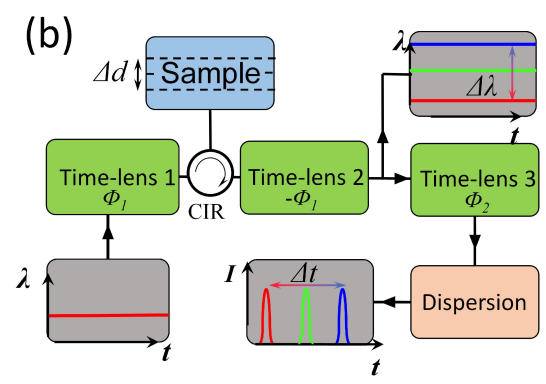

Fig.1 Principle of PASTA-based WET system: (a) schematic ray diagram; (b) simplified setup 
The magnification factor can be calculated as $M=\Phi_{2} / \Phi_{I}=68.4$, and the current depth resolution is $180 \mu \mathrm{m}$. The depth-resolving performance is mainly limited by the FWM conversion bandwidth of the highly-nonlinear fiber (HNLF). By using dispersion-engineered silicon waveguide, resolution less than $20 \mu \mathrm{m}$ is achievable [4]. The 60$\mathrm{MHz}$ A-scan rate is determined by the repetition rate of the swept pump in the time-lenses, which has great flexibility according to the design. Sensitivity of the system, which is limited by the FWM efficiency, is currently $40 \mathrm{~dB}$. Comparing to the conventional temporal-magnification systems, the PASTA-based WET system is more suitable for bio-imaging. Instead of fs-pulse-illumination, the first time-lens generates a long chirped pulse to avoid the potential damage as well as the nonlinearity inside the bio-sample.

The sample under test consisted of a $200-\mu \mathrm{m}$-thick and a 1-mm-thick glass layer, as shown in Fig. 2(a). The images produced by both OSA-based WET system and PASTA-based WET system were obtained by moving the glass sample over $5 \mathrm{~mm}$ by a transition stage. As can be observed from Fig. 2(b), the OSA-based WET system could not resolve the thin glass layer, as the $0.05 \mathrm{~nm}$ spectral resolution of OSA resulted in only $300-\mu \mathrm{m}$ depth resolution with the current WET system. In Fig. 2(c) however, the two-layer structure could be clearly observed in the PASTAbased WET system even at 60-MHz A-scan rate. Each A-scan produced a trace like Fig. 2(d), which was the magnified waveform according to the time delays. Under the current magnification factor and the A-scan rate, the time-delay of 1-mm depth only occupies about 330ps over the $16 \mathrm{~ns}$ output window. Owing to the trade-off between the repetition rate and the length of the output window, the A-scan rate can be further boosted to $\mathrm{GHz}$ regime using a shorter output window.
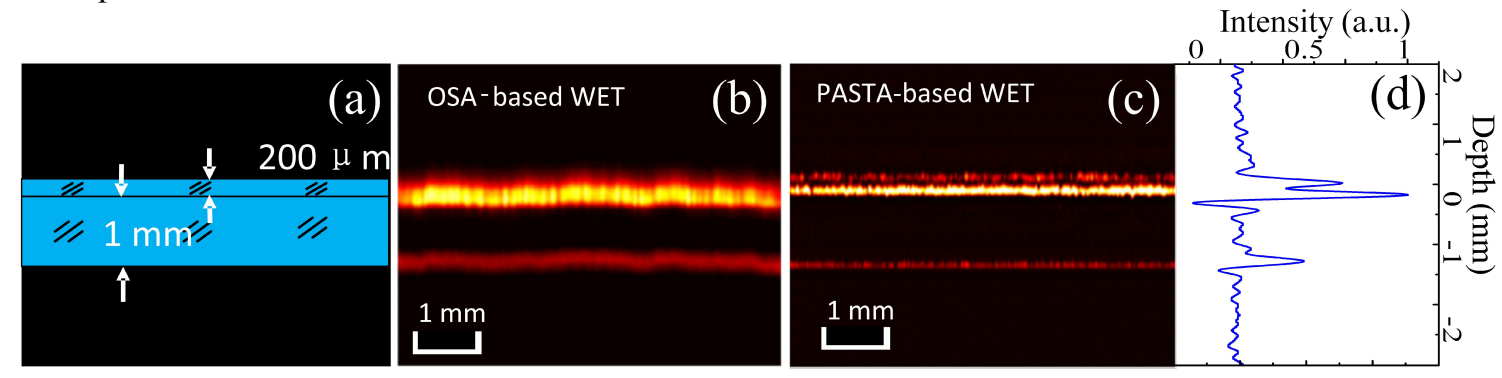

Fig.2 Imaging results: (a) schematic diagram of glass sample; (b) image generated by OSA-based WET system; (c) image generated by PASTAbase WET system; (d) waveform of a single A-scan in PASTA-based WET system.

\section{Conclusion}

In summary, by integrating a third time-lens into the WET system, we achieved ultrafast operation where temporal magnification is for the first time applied to cross-sectional imaging. By performing depth-wavelength-time mapping, the A-scan rate is increased from $5 \mathrm{~Hz}$ to $60 \mathrm{MHz}$ with depth resolution of $180 \mu \mathrm{m}$. By utilizing better nonlinear media, depth resolution can be improved to less than $20 \mu \mathrm{m}$, which will definitely make the PASTA-based WET system a promising member in the family of tomography modalities.

\section{Acknowledgement}

This work was partially supported by grant from the Research Grants Council of the Hong Kong Special Administrative Region, China (Project Nos. HKU 7172/12 E and HKU 17208414 E). The authors also acknowledge Sumitomo Electric Industries for providing the HNL-DSF and Alnair for providing the variable bandwidth tunable band-pass filter (VBTBPF).

\section{References}

[1] D. Huang, E. A. Swanson, C. P. Lin, J. S. Schuman, W. G. Stinson, W.Chang, M. R. Hee, T. Flotte, K. Gregory, C. A. Puliafito, and J. G. Fujimoto, “Optical coherence tomography," Science 254, 1178-1181 (1991).

[2] C. Zhang and K. K. Y. Wong, "Wavelength-encoded tomography based on optical temporal Fourier transform," Appl. Phys. Lett. 105, 091109 (2014).

[3] C. Zhang, J. Xu, P. C. Chui, and K. K. Y. Wong, "Parametric spectro-temporal analyzer (PASTA) for real-time optical spectrum observation," Sci. Rep. 3, 2064 (2013).

[4] R. Salem, M. A. Foster, A. C. Turner-Foster, D. F. Geraghty, M. Lipson, and A. L. Gaeta, "High-speed optical sampling using a silicon-chip temporal magnifier," Opt. Express 17, 4324-4329 (2009).

[5] V. J. Hernandez, C. V. Bennett, B. D. Moran, A. D. Drobshoff, D. Chang, C. Langrock, M. M. Fejer and M. Ibsen, "104 MHz rate single-shot recording with subpicosecond resolution using temporal imaging," Opt. Express 21, 196-203 (2013). 